\title{
Fault Detection of A Sandwich System with Dead-zone Based on Robust Observer
}

\author{
Zupeng Zhou ${ }^{1 *}$, Yonghong $\operatorname{Tan}^{2}$, Peng $\mathrm{Shi}^{3}$ \\ 1. School of Mechanical and Electrical Engineering \\ Guilin University of Electronic Technology, Guilin, China; Email: zhouzupeng@ guet.edu.cn \\ 2. College of Information, Mechanical and Electrical Engineering \\ Shanghai Normal University, Shanghai 200234, China \\ 3. School of Electrical and Electronic Engineering, University of Adelaide, Adelaide, SA 5005 \\ Australia
}

\begin{abstract}
Non-smooth sandwich systems with dead-zone widely exist in the real engineering applications. For accurately detecting its faults, a novel robust observer has been proposed in this paper. With the consideration of the model uncertainties, disturbances, and switching error which specially belongs to the system, the so-called general disturbance is defined. After that, the conventional dynamic robust observer design method can be applied to the system. Then, for saving the computing time and effectively reducing the effect of the disturbances to the residual, the main frequencies of the disturbances have been identified by the spectrum analysis of the residual created by the conventional observer and the zeros assignment methodology has been applied to get one feedback matrix of the robust observer. Finally, the rest of the feedback matrices of the robust observer can be obtained by solving an optimal problem with $H_{\infty, F} / H_{-, F}$ as the minimizing object. For verifying the effectiveness of this novel robust observer, the comparison between the proposed robust non-smooth scheme and the conventional method has been made. The final results show that the proposed robust fault detection approach can detect the actuator and sensor faults of the system more accurately and timely with a lower missing and false alarm rates comparing with the conventional one.
\end{abstract}

Key words: Robust observer; dead-zone; sandwich system; general disturbance; fault detection

\section{Introduction}

A dead zone is a non-smooth and nonlinear character which widely exists in all kinds of motors, mechanical transition systems, hydraulic systems, and mechatronic systems [1]. Dead zone usually does not exist independently. On the contrary, it usually connects with other parts. For instance, in a DC motor servo system, the linear parts of the power amplifier and the DC motor can be regarded as the front linear subsystem while the load of the motor can be regarded as the back linear subsystem. The dead zone of the DC motor embedded between the two dynamic linear parts and this structure can be regarded as sandwich systems with dead zone. In the industry field, many systems can be described as sandwich systems with dead zone such as a hydraulic actuator of aircraft lift [2], position stage 
driven by a DC motor, and a hydraulic actuator controlled by a pilot valve etc. [3].

In engineering practice, the dead zone does not exist independently and it usually connects with other conventional parts. If the dead zone nonlinearity is sandwiched into two linear dynamic subsystems, this system can be defined as the sandwich system with dead zone. In real application, the model uncertainties and disturbances always exist and how to design a robust fault prediction observer to restrain the effect of the model uncertainties and disturbances are of crucial importance [4-5]. A Luenberger-type switching observer is designed for a class of hybrid linear systems [6-7], while an observer is proposed for a class of piecewise affine systems, respectively in [8-9].

In [10], a novel fault detection and identification (FDI) scheme is presented for a class of nonlinear systems with model uncertainty. The heart of this approach is an on-line approximator, referred to as fault tracking approximator (FTA). In [11], a new sensor fault detection, isolation, and identification (FDII) strategy is proposed using the multiple-model (MM) approach. The scheme is based on multiple hybrid Kalman filters (MHKFs), which represents an integration of a nonlinear mathematical model of the system with a number of piecewise linear (PWL) models. The work in [12] investigates a fault detection and accommodation (FDA) problem of saturated actuators for trajectory tracking of underactuated surface vessels (USVs) in the presence of nonlinear uncertainties. In [13], a new strategy for integration of fault estimation within fault-tolerant control is proposed. A new delay-derivative-dependent sliding mode observer (SMO) design is given in [14] for a class of linear uncertain time-varying delay systems is presented. In [15], the problem of fault detection for discrete-time Lipschitz nonlinear systems with additive white Gaussian noise channels subject to signal-to-noise ratio constraints is investigated. A unknown input observer-based robust fault estimation for systems corrupted by partially decoupled disturbances is proposed, and the effectiveness of this method is verified by simulations [16]. In [17], both the steering angle and sideslip angle of a vehicle can be estimated by using an unknown input observer as well. In the unknown input observer, all the model uncertainties, disturbances, and even faults can be regarded as the unknown input in this observer. From the above literature review, it is clear that many works focusing on observer designs for specific non-linear systems have been done in recent years by using a multiple-model approach, a hybrid Kalman filter, a sliding mode observer, and an unknown observer.

Note that the work on a state-estimation of the sandwich system with dead zone, backlash, and hysteresis, has been conducted respectively [18-20]. Recently, an observer to realize the more accurate fault detection of a mechanical system with inherent backlash without considering the model uncertainties and disturbances is designed in [21].

However, the sandwich system with dead zone not only has the non-smooth and nonlinear part which connects with the front and back linear parts but also has the immeasurable interval variables i.e., the input $y_{1}(k)$ and output $v(k)$ of the dead zone. In other words, only the input variable $u(k)$ and the output variable $y_{2}(k)$ are measurable (See in Fig.1). In addition, in the robust fault detection 
observer design process, the model uncertainties and disturbances have to be considered. Therefore, this kind of system is much more complicated than the traditional ones and accurate fault prediction can be very challenging. Till now, from the authors' best knowledge, the problem of observers design to deal with robust fault prediction for the sandwich system with dead zone yet has not been fully investigated, which motivates our current work.

\section{Model of the sandwich system with dead zone}

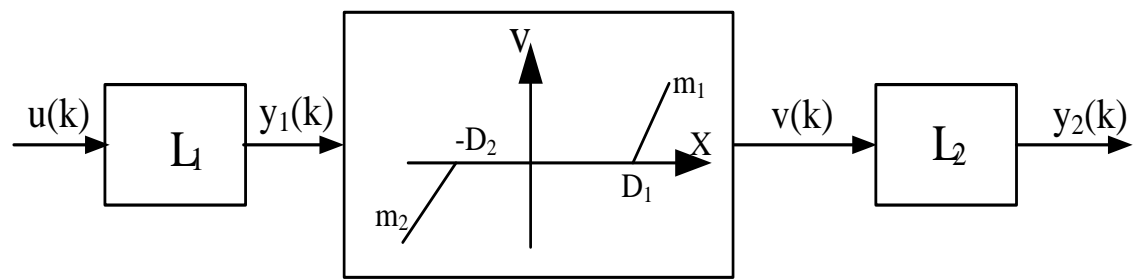

Fig.1 The structure of the sandwich systems with dead zone

A typical sandwich system with dead zone and the corresponding architecture of this system is shown in Fig.1, where, $u(k)$ and $y_{2}(k)$ are the measurable input and output of the system, respectively. $y_{1}(k)$ and $v(k)$ are the interval variables which cannot be measured directly. $\mathrm{L}_{1}($.$) is$ the front linear subsystem and $\mathrm{L}_{2}($.$) is the back linear subsystem.$

The front linear subsystem $L_{1}($.$) can be described as$

$\left\{\begin{array}{l}\mathbf{x}_{1}(k+1)=\mathbf{A}_{1} \mathbf{x}_{1}(k)+\mathbf{B}_{1} u(k) \\ y_{1}(k)=\mathbf{C}_{1} \mathbf{x}_{1}(k)\end{array}\right.$

and the back linear subsystem $\mathrm{L}_{2}($.) can be described as

$\left\{\begin{array}{l}\mathbf{x}_{2}(k+1)=\mathbf{A}_{2} \mathbf{x}_{2}(k)+\mathbf{B}_{2} v(k) \\ y_{2}(k)=\mathbf{C}_{2} \mathbf{x}_{2}(k)\end{array}\right.$

where $\mathbf{x}_{i} \in \mathbf{R}^{n_{i} \times 1}, \quad \mathbf{A}_{i} \in \mathbf{R}^{n_{i} \times n_{i}}, \mathbf{B}_{i} \in \mathbf{R}^{n_{i} \times 1}, \quad y_{i} \in R^{1 \times 1}, \mathbf{C}_{i} \in \mathbf{R}^{1 \times n_{i}}, u \in R^{1 \times 1}, v \in R^{1 \times 1}$, and $i=1,2$.

Here, $x_{1 i}$ and $x_{2 i}$ represent the $i$ th state variable of $\mathrm{L}_{1}$ and $\mathrm{L}_{2}$, respectively. $\mathbf{A}_{i} \in \mathbf{R}^{n_{i} \times n_{i}}$ is the state transition matrix, $\mathbf{B}_{i} \in \mathbf{R}^{n_{i} \times 1}$ is the input matrix, $y_{2} \in R^{1 \times 1}$ is the output variable of the whole system, $n_{i}$ represents the dimension of the $i$ th linear subsystem, $u \in R^{1 \times 1}$ is the input variable, $y_{1} \in R^{1 \times 1}$ is the input variable of the dead zone and $v \in R^{1 \times 1}$ is the output variable of the dead zone. Without the loss generality, in the state-space function, for $\mathrm{L}_{1}$ and $\mathrm{L}_{2}$, we set $x_{1 n_{1}}(k)=y_{1}(k)$ and 
$x_{2 n_{2}}(k)=y_{2}(k)$, respectively.

Based on reference [2] and [3] as well as the property of the dead zone in middle part of Fig.1, the model of the dead zone can be obtained as follows.

Define $m(k)$ and $v_{1}(k)$, respectively, as the imposed variables, i.e.,

$$
\begin{aligned}
& m(k)=m_{1}+\left(m_{2}-m_{1}\right) h(k), \\
& v_{1}(k)=m(k)\left(y_{1}(k)-D_{1} h_{1}(k)+D_{2} h_{2}(k)\right),
\end{aligned}
$$

where, $h(k)=\left\{\begin{array}{ll}1, & y_{1}(k)<0 \\ 0, & \text { else }\end{array}, h_{1}(k)=\left\{\begin{array}{ll}1, & y_{1}(k)>D_{1} \\ 0, & \text { else }\end{array}\right.\right.$, and $h_{2}(k)=\left\{\begin{array}{ll}1, & y_{1}(k)<-D_{2} \\ 0, & \text { else }\end{array}\right.$ are the switch functions which are used to judge and switch the operation zones, i.e., the linear zone and the dead zone. Based on the properties of dead zone, it yields

$\breve{v}(k)=v_{1}(k)-h_{3}(k) v_{1}(k)=\left(1-h_{3}(k)\right) v_{1}(k)$

where, $h_{3}(k)=\left\{\begin{array}{ll}1, & h_{1}(k)+h_{2}(k)=0 \\ 0, & h_{1}(k)+h_{2}(k)=1\end{array}\right.$ is the switch function utilized to separate the linear zones from the dead zone.

Based on (5), when $h_{3}(k)=0$ the system operates on linear zone and $v(k)=v_{1}(k)$. When $h_{3}(k)=1$ the system operates on the dead zone and $v(k)=v_{1}(k)-v_{1}(k)=0$.

Substituting (4) into (5) results in

$$
\begin{aligned}
& \nu(k)=\left(1-h_{3}(k)\right) v_{1}(k)=\left(1-h_{3}(k)\right) m(k)\left(y_{1}(k)-D_{1} h_{1}(k)+D_{2} h_{2}(k)\right) \\
& \left.=\left(1-h_{3}(k)\right) m(k) y_{1}(k)-\left(1-h_{3}(k)\right) m(k) D_{1} h_{1}(k)+\left(1-h_{3}(k)\right) m(k) D_{2} h_{2}(k)\right) .
\end{aligned}
$$

By substituting (6) into (3) and noticing $y_{1}(k)=x_{1 n_{1}}(k)$, it results in

$$
\begin{aligned}
& \mathbf{x}_{2}(k+1)=A_{22} \mathbf{x}_{2}(k+1)+B_{22} v(k)=A_{22} \mathbf{x}_{2}(k+1) \\
& \left.+B_{22}\left[\left(1-h_{3}(k)\right) m(k) x_{1 n_{1}}(k)-\left(1-h_{3}(k)\right) m(k) D_{1} h_{1}(k)+\left(1-h_{3}(k)\right) m(k) D_{2} h_{2}(k)\right)\right] .
\end{aligned}
$$

Based on (1), (2), and (7), it leads to

$$
\left\{\begin{array}{l}
{\left[\begin{array}{l}
\mathbf{x}_{1}(k+1) \\
\mathbf{x}_{2}(k+1)
\end{array}\right]=\left[\begin{array}{ll}
\mathbf{A}_{1} & \mathbf{0} \\
\mathbf{A}_{21 i} & \mathbf{A}_{2}
\end{array}\right]\left[\begin{array}{l}
\mathbf{x}_{1}(k) \\
\mathbf{x}_{2}(k)
\end{array}\right]+\left[\begin{array}{l}
\mathbf{B}_{1} \\
\mathbf{0}
\end{array}\right] u(k)+\left[\begin{array}{l}
\mathbf{0} \\
\boldsymbol{\theta}_{22 i}
\end{array}\right]} \\
y(k)=\mathbf{C x}(k)
\end{array}\right.
$$

where $\quad \mathbf{A}_{21 i}=\left[\begin{array}{ll}\boldsymbol{\beta}_{1} & \boldsymbol{\beta}_{2 i}\end{array}\right] \quad, \quad \boldsymbol{\beta}_{1}=\mathbf{0} \in \mathbf{R}^{n_{2} \times\left(n_{2}-1\right)} \quad, \quad \boldsymbol{\beta}_{2 i}=\left\{\begin{array}{l}\mathbf{B}_{2} m_{1}(k), i=1 \\ \mathbf{0}, i=2 \\ \mathbf{B}_{2} m_{2}(k), i=3\end{array} \quad, \quad \boldsymbol{\beta}_{2 i} \in \mathbf{R}^{n_{2} \times 1}\right.$, 


$$
\boldsymbol{\theta}_{22 i}=\left\{\begin{array}{l}
-\mathbf{B}_{2} m_{1}(k) D_{1}, i=1 \\
\mathbf{0}, i=2, \quad \boldsymbol{\theta}_{22 i} \in \mathbf{R}^{n_{2} \times 1}, \text { and } \mathbf{x}(k)=\left[\begin{array}{l}
\mathbf{x}_{1}(k) \\
\mathbf{x}_{2}(k)
\end{array}\right] \in \mathbf{R}^{\left(n_{1}+n_{2}\right) \times 1} .
\end{array}\right.
$$

The index of the operation zone is defined as $i=\left\{\begin{array}{ll}1, & x_{1 n_{1}}>D_{1}(\text { linear increasing zone }) \\ 2, & -D_{2} \leq x_{1 n_{1}}(k) \leq D_{1}(\text { dead zone }) \\ 3, & x_{1 n_{1}}(k)<-D_{2}(\text { linear decreasing zone })\end{array}\right.$.

Because only the output $y_{2}(k)$ of the sandwich system with dead zone described in section 2 of Fig.1 can be measurable, then we can obtain that $\mathbf{C}=[0,0, \ldots, 0,1] \in \mathbf{R}^{1 \times\left(n_{1}+n_{2}\right)}$. For the convenience of proof and block matrix calculation, matrix $\mathbf{C}$ can be blocked as $\mathbf{C}=\left[\begin{array}{ll}\mathbf{C}_{11} & \mathbf{C}_{22}\end{array}\right]$ where $\mathbf{C}_{11}=[0,0, \ldots, 0]=\mathbf{0} \in \mathbf{R}^{1 \times n_{1}}$ and $\mathbf{C}_{22}=[0,0, \ldots, 1] \in \mathbf{R}^{1 \times n_{2}}$.

In (8), $\mathbf{0}$ represents the zero matrixes with appropriate dimension.

If we set $\mathbf{A}(k)=\left[\begin{array}{ll}\mathbf{A}_{1} & \mathbf{0} \\ & \\ \mathbf{A}_{21 i} & \mathbf{A}_{2}\end{array}\right], \quad \mathbf{B}=\left[\begin{array}{l}\mathbf{B}_{1} \\ \mathbf{0}\end{array}\right]$, and $\boldsymbol{\eta}(k)=\left[\begin{array}{l}\mathbf{0} \\ \boldsymbol{\theta}_{22 i}\end{array}\right] \quad(i=1,2,3), \quad$ equation (8) can be rewritten as

$\mathbf{x}(k+1)=\mathbf{A}(k) \mathbf{x}(k)+\mathbf{B} u(k)+\boldsymbol{\eta}(k)$

where $\mathbf{A}(k)$ is the transition matrix of different operation zone, $\mathbf{B}$ is the input matrix, and $\boldsymbol{\eta}(k)$ is the switch vector caused by the existence of dead zone.

\section{Robust fault prediction observer design for the sandwich system with dead zone}

By the state space equation of eq. (9) and taking the model uncertainty, disturbances, and faults into consideration, it leads to

$\left\{\begin{array}{l}\mathbf{x}(k+1)=\mathbf{A}(k) \mathbf{x}(k)+\mathbf{B} u(k)+\boldsymbol{\eta}(k)+\mathbf{B}_{d} \mathbf{d}(k)+\mathbf{B}_{f} \mathbf{f}(k) \\ y(k)=\mathbf{C x}(k)+\mathbf{D}_{d} \mathbf{d}(k)+\mathbf{D}_{f} \mathbf{f}(k)\end{array}\right.$,

Where, $\mathbf{d}(k) \in \mathbf{R}^{r \times 1}$ is the disturbance vector which includes the model uncertainty and disturbance. $\mathbf{B}_{d} \in \mathbf{R}^{n \times r}$ is the disturbance input matrix which determines how each disturbance affects the normal state variables, $\mathbf{D}_{d} \in \mathbf{R}^{1 \times r}$ is the disturbance output matrix which determines how each disturbance affects the output of the normal system. $\mathbf{f}(k) \in \mathbf{R}^{f \times 1}$ is the fault vector (including the actuator fault and sensor fault). $\mathbf{B}_{f} \in \mathbf{R}^{n \times f}$ is the fault input matrix and $\mathbf{D}_{f} \in \mathbf{R}^{1 \times f}$ is the fault output matrix. 


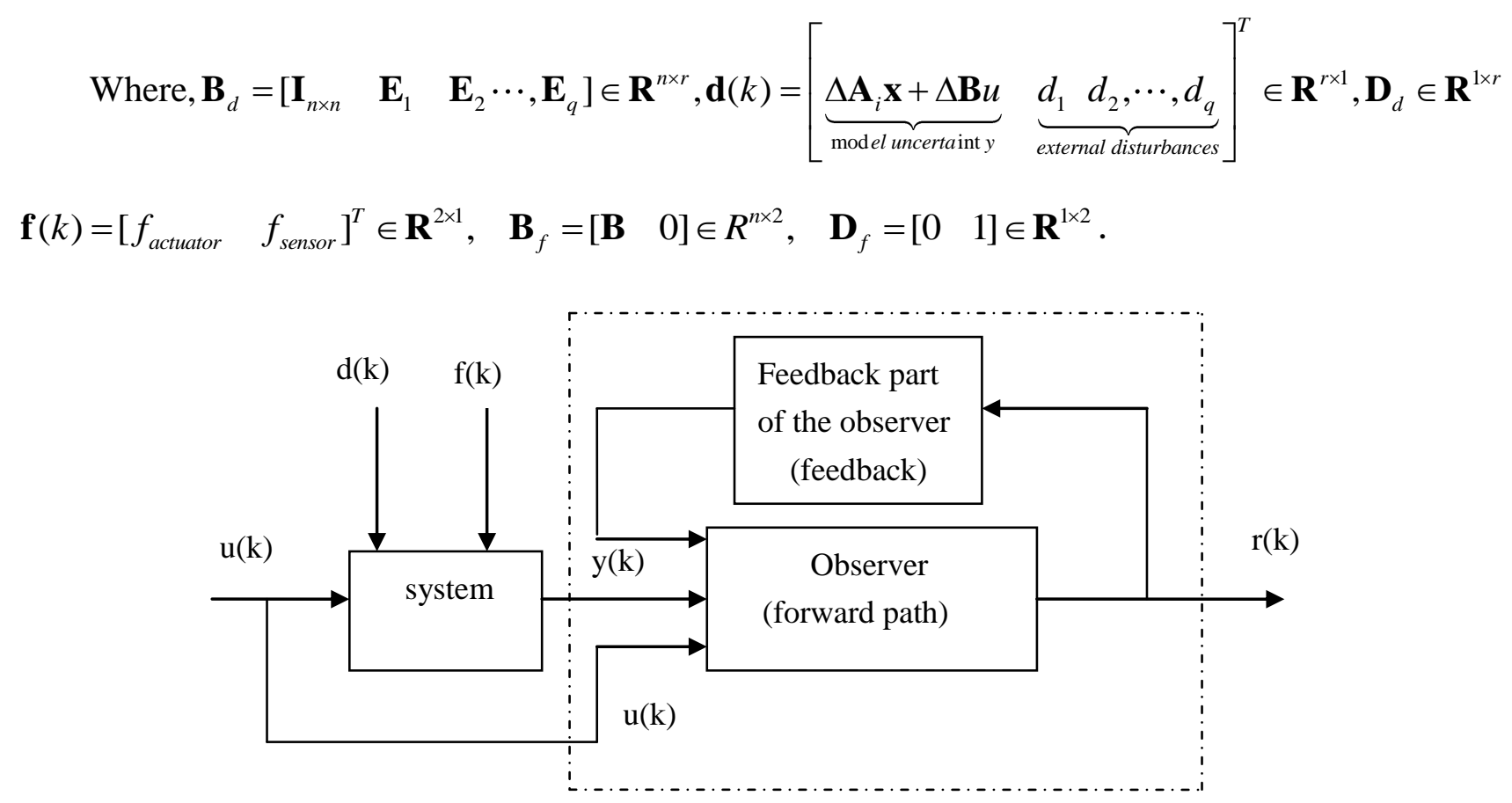

Fig.2 Structure of the dynamic robust observer

The dynamic robust observer is constructed as in Fig.2. $\mathbf{A}_{l}$ is the transition matrix of the system which assuming the dead zone does not exist. When the system operates on this assuming zone we called it a basic operation zone. In other words, $\mathbf{A}_{l}$ is the transition matrix of the system if the dead zone is regarded as a proportional linear part and this operation zone called the basic operation zone. The equivalent coefficient is defined as $C_{l}$. Therefore, the dynamical robust observer can be described as eq. (11).

$$
\left\{\begin{array}{l}
\left\{\begin{array}{l}
\mathbf{z}_{1}(k+1)=\mathbf{K}_{1} \mathbf{z}_{1}(k)+\mathbf{K}_{2} r(k) \\
\mathbf{v}(k+1)=\mathbf{K}_{3} \mathbf{z}_{1}(k+1)+\mathbf{K}_{4} r(k+1)
\end{array}\right. \\
\left\{\begin{array}{l}
\hat{\mathbf{x}}(k+1)=\mathbf{A}_{l} \hat{\mathbf{x}}(k)+\mathbf{B} u(k)+\mathbf{v}(k) \\
\hat{y}(k+1)=\mathbf{C} \hat{\mathbf{x}}(k+1)
\end{array}\right.
\end{array}\right.
$$

Where, $\quad \mathbf{A}_{l}=\left[\begin{array}{cc}\mathbf{A}_{1} & \mathbf{0} \\ \mathbf{A}_{211} & \mathbf{A}_{2}\end{array}\right], \quad A_{211}$ is the block matrix of the system when the system operates on the basic operation zone. The residual is $r(k)=y(k)-\hat{y}(k)$ and $\mathbf{z}_{1}(k) \in \mathbf{R}^{n \times 1}$ is the dynamical feedback state variables, and $\mathbf{v}(k) \in \mathbf{R}^{n \times 1}$ is the output of the feedback dynamical part. $\mathbf{K}_{1} \in R^{n \times n}, \mathbf{K}_{2} \in R^{n \times 1}, \mathbf{K}_{3} \in R^{n \times n}, \mathbf{K}_{4} \in R^{n \times 1}$ are the feedback matrices which need to be designed for the robust observer.

By setting $\mathbf{e}(k)=\mathbf{x}(k)-\hat{\mathbf{x}}(k)$ and taking the system described in eq. (10) and the robust observer 
described in eq. (11) into consideration, the observer described in eq. (11) with an expanded vector form and its expanded dynamic error can be described in eq. (12) and eq. (13), respectively.

$\left\{\begin{array}{l}{\left[\begin{array}{l}\hat{\mathbf{x}}(k+1) \\ \mathbf{z}_{1}(k+1)\end{array}\right]=\left[\begin{array}{ll}\mathbf{A}_{l}-\mathbf{K}_{4} \mathbf{C} & \mathbf{K}_{3} \\ -\mathbf{K}_{2} \mathbf{C} & \mathbf{K}_{1}\end{array}\right]\left[\begin{array}{l}\hat{\mathbf{x}}(k) \\ \mathbf{z}_{1}(k)\end{array}\right]+\left[\begin{array}{l}\mathbf{B} \\ \mathbf{0}\end{array}\right] u(k)+\left[\begin{array}{l}\mathbf{K}_{4} \\ \mathbf{K}_{2}\end{array}\right] y(k)} \\ \hat{y}(k+1)=\mathbf{C} \hat{\mathbf{x}}(k+1)\end{array}\right.$

If we set $\xi(k+1)=\left[\begin{array}{c}\mathbf{e}(k+1) \\ \mathbf{z}_{1}(k+1)\end{array}\right]$, it leads to

$$
\left\{\begin{array}{l}
\boldsymbol{\xi}(k+1)=\left[\begin{array}{ll}
\mathbf{A}_{l}-\mathbf{K}_{4} \mathbf{C} & -\mathbf{K}_{3} \\
\mathbf{K}_{2} \mathbf{C} & \mathbf{K}_{1}
\end{array}\right] \boldsymbol{\xi}(k)+\left[\begin{array}{l}
\mathbf{B}_{d^{*}}^{*}-\mathbf{K}_{4} \mathbf{D}_{d^{*}} \\
\mathbf{K}_{2} \mathbf{D}_{d^{*}}
\end{array}\right] \mathbf{d}^{*}(k)+\left[\begin{array}{l}
\mathbf{B}_{f}-\mathbf{K}_{4} \mathbf{D}_{f} \\
\mathbf{K}_{2} \mathbf{D}_{f}
\end{array}\right] \mathbf{f}(k) . \\
\mathbf{e}(k)=\left[\begin{array}{ll}
\mathbf{I}_{n \times n} & \mathbf{0}_{n \times m}
\end{array}\right] \boldsymbol{\xi}(k)+\mathbf{D}_{f} \mathbf{f}(k)
\end{array}\right.
$$

In eq. (13), $\mathbf{B}_{d^{*}}^{*}$ represents the expanded disturbance input matrix and $\mathbf{D}_{d^{*}}$ represents the expanded disturbance output matrix, and $\mathbf{d}^{*}(k)$ is the expanded disturbance vector. $\mathbf{d}^{*}(k)$ derives from d by adding the switching error which caused by the wrong zone estimation into the disturbance vector. Therefore, we call it expanded disturbance vector.

The detail expressions of these matrices are shown as below.

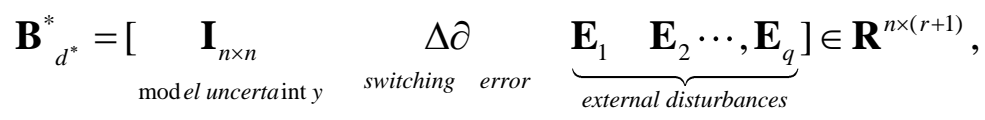

$$
\begin{aligned}
& \mathbf{d}^{*}(k)=[\underbrace{\Delta \mathbf{A}(k) \mathbf{x}+\Delta \mathbf{B} u}_{\text {model uncertaint } y} \quad \delta_{x} \quad \underbrace{d_{1} d_{2}, \cdots, d_{q}}_{\text {switching error }}]^{T} \in \mathbf{R}^{(r+1) \times 1}, \mathbf{D}_{d^{*}} \in \mathbf{R}^{1 \times(r+1)},
\end{aligned}
$$

Especially, here $\Delta \partial=\left[\begin{array}{l}\mathbf{0} \\ \mathbf{B}_{22}\end{array}\right] \in \mathbf{R}^{n \times 1}$ represents the input disturbance matrix caused by operation zone mismatching and $\delta_{x} \in R^{1 \times 1}$ represents the input disturbance vector caused by the operation zone mismatching.

Eq. (14) can be obtained by redefining the symbols in eq. (13). By these redefinitions, eq. (14) is more concise comparing with eq. (13).

$$
\left\{\begin{array}{l}
\tilde{\mathbf{x}}(k+1)=\tilde{A} \tilde{\mathbf{x}}(k)+\tilde{B}_{d^{*}}^{*} \mathbf{d}^{*}(k)+\tilde{B}_{f} \mathbf{f}(k) \\
\mathbf{r}(k)=\tilde{C} \tilde{\mathbf{x}}(k)+\tilde{D}_{d^{*}} \mathbf{d}^{*}(k)+\tilde{D}_{f} \mathbf{f}(k)
\end{array}\right.
$$

where, $\tilde{\mathbf{x}}(k)=\left[\begin{array}{l}\mathbf{e}(k) \\ \mathbf{z}_{1}(k)\end{array}\right], \tilde{\mathbf{A}}=\left[\begin{array}{ll}\mathbf{A}_{l}-\mathbf{K}_{4} \mathbf{C} & -\mathbf{K}_{3} \\ \mathbf{K}_{2} \mathbf{C} & \mathbf{K}_{1}\end{array}\right], \quad \tilde{\mathbf{B}}_{d^{*}}^{*}=\left[\begin{array}{l}\mathbf{B}_{d^{*}}^{*}-\mathbf{K}_{4} \mathbf{D}_{d^{*}} \\ \mathbf{K}_{2} \mathbf{D}_{d^{*}}\end{array}\right], \quad \tilde{\mathbf{B}}_{f}=\left[\begin{array}{l}\mathbf{B}_{f}-\mathbf{K}_{4} \mathbf{D}_{f} \\ \mathbf{K}_{2} \mathbf{D}_{f}\end{array}\right]$,

$$
\tilde{\mathbf{C}}=\left[\begin{array}{ll}
\mathbf{C} & \mathbf{0}
\end{array}\right], \tilde{\mathbf{D}}_{d^{*}}=\mathbf{D}_{d^{*}}, \quad \tilde{\mathbf{D}}_{f}=\mathbf{D}_{f} .
$$

After making $\mathrm{Z}$ transform of eq. (14), it leads to eq. (15)

$$
\mathbf{r}(z)=\tilde{G}_{d^{*} r}\left(\tilde{\mathbf{A}}, \mathbf{K}_{d}\right) \mathbf{d}^{*}(z)+\tilde{G}_{f r}\left(\tilde{\mathbf{A}}, \mathbf{K}_{d}\right) \mathbf{f}(z) .
$$


By eq. (15), the residual is not only related to the fault but also to the general disturbances. Therefore, the purpose of designing the robust fault detection observer is to select an appropriate feedback matrices (i.e., $\mathbf{K}_{d}=\left[\mathbf{K}_{1}, \mathbf{K}_{2}, \mathbf{K}_{3}, \mathbf{K}_{4}\right]$ ) that is sensitive to the fault and not sensitive to the general disturbances.

The transfer function of $\mathbf{d}^{*}(k)$ to $\mathbf{r}(k)$ is $G_{d^{*} r}\left(\tilde{\mathbf{A}}, \mathbf{K}_{d}\right)$.

$$
\tilde{G}_{d^{*} r}\left(\tilde{\mathbf{A}}, \mathbf{K}_{d}\right)=\frac{\mathbf{r}(z)}{\mathbf{d}^{*}(z)}=\tilde{\mathbf{C}}(\mathbf{I} z-\tilde{\mathbf{A}})^{-1} \tilde{\mathbf{B}}_{d^{*}}+\tilde{\mathbf{D}}_{d^{*}}
$$

The transfer function of $\mathbf{f}(k)$ to $\mathbf{r}(k)$ is $G_{\mathbf{f r}}\left(\tilde{A}, K_{d}\right)$.

$$
\tilde{G}_{f r}(\tilde{\mathbf{A}}, \mathbf{K})=\frac{\mathbf{r}(z)}{\mathbf{f}(z)}=\tilde{\mathbf{C}}(\mathbf{I} z-\tilde{\mathbf{A}})^{-1} \tilde{\mathbf{B}}_{f}+\tilde{\mathbf{D}}_{f}
$$

Where, $\tilde{\mathbf{A}}=\left[\begin{array}{cc}\mathbf{A}_{l}-\mathbf{K}_{4} \mathbf{C} & -\mathbf{K}_{3} \\ \mathbf{K}_{2} \mathbf{C} & \mathbf{K}_{1}\end{array}\right], \quad \tilde{\mathbf{B}}_{d^{*}}^{*}=\left[\begin{array}{l}\mathbf{B}_{d^{*}}^{*}-\mathbf{K}_{4} \mathbf{D}_{d^{*}} \\ \mathbf{K}_{2} \mathbf{D}_{d^{*}}\end{array}\right], \quad \tilde{\mathbf{C}}=\left[\begin{array}{ll}\mathbf{I}_{n \times n} & \mathbf{0}_{n \times m}\end{array}\right], \quad \tilde{\mathbf{D}}_{d^{*}}=\mathbf{D}_{d^{*}}$.

Then, the switching error caused by the wrong operation zone of the observer has been analyzed as below. At the earlier stage, the variable which determines the operation zone of the sandwich system can be different from the real value. Therefore, the observer may operate on the wrong zone at the earlier stage and make the estimation error. By eq. (9), this error is $\Delta \mathbf{A}_{1}(k) \mathbf{x}(k)+\Delta \boldsymbol{\eta}$ in which $\Delta \mathbf{A}_{1}(k)=\mathbf{A}(k)-\mathbf{A}_{l}$ and $\Delta \boldsymbol{\eta}=\boldsymbol{\eta}(k)-\mathbf{0} . \quad$ This switching error can be regarded as a general disturbance and it can be expressed as below.

$$
\begin{aligned}
& \Delta \mathbf{A}_{1}(k) \mathbf{x}(k)+\Delta \boldsymbol{\eta}=\left(\mathbf{A}(k)-\mathbf{A}_{l}\right) \mathbf{x}+\boldsymbol{\eta}(k)=\left(\left[\begin{array}{ll}
\mathbf{A}_{1} & \mathbf{0} \\
\mathbf{A}_{21}(k) & \mathbf{A}_{22}
\end{array}\right]-\left[\begin{array}{ll}
\mathbf{A}_{11} & \mathbf{0} \\
\mathbf{A}_{211} & \mathbf{A}_{22}
\end{array}\right]\right) \mathbf{x}+\left[\begin{array}{l}
\mathbf{0} \\
\boldsymbol{\theta}_{22}(k)
\end{array}\right] \\
& =\left[\begin{array}{ll}
\mathbf{0} & \mathbf{0} \\
\mathbf{A}_{21}(k)-\mathbf{A}_{211} & \mathbf{0}
\end{array}\right]\left[\begin{array}{l}
\mathbf{x}_{1}(k) \\
\mathbf{x}_{2}(k)
\end{array}\right]+\left[\begin{array}{l}
\mathbf{0} \\
\boldsymbol{\theta}_{22}(k)
\end{array}\right]=\left[\begin{array}{l}
\mathbf{0} \\
\Delta \mathbf{A}_{2}(k) \mathbf{x}_{1}(k)+\boldsymbol{\theta}_{22}(k)
\end{array}\right]
\end{aligned}
$$

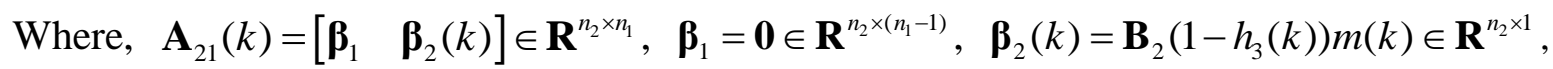

$$
\begin{aligned}
& \mathbf{A}_{211}=\left[\begin{array}{ll}
\boldsymbol{\beta}_{1} & \boldsymbol{\beta}_{2 l}
\end{array}\right] \in \mathbf{R}^{n_{2} \times n_{1}}, \boldsymbol{\beta}_{2 l}=\mathbf{B}_{2} \mathbf{C}_{l} \in \mathbf{R}^{n_{2} \times 1}
\end{aligned}
$$

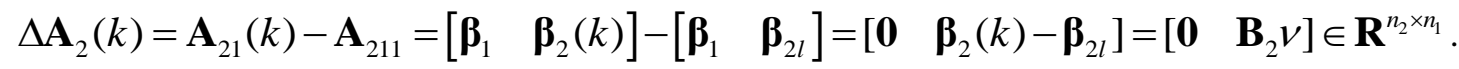

By setting $v=\left(1-h_{3}(k)\right) m(k)-C_{l} \in R^{1 \times 1}$ it leads to

$$
\begin{aligned}
& \left(\mathbf{A}_{21}(k)-\mathbf{A}_{211}\right) \mathbf{x}_{1}(k)=\left[\begin{array}{ll}
\mathbf{0} & \mathbf{B}_{2} v
\end{array}\right] \mathbf{x}_{1}(k)=\mathbf{B}_{2} v x_{1 n_{1}} \in \mathbf{R}^{n_{2} \times 1} \text {. Since } \\
& \left.\boldsymbol{\theta}_{22}(k)=-\mathbf{B}_{2}\left[\left(1-h_{3}(k)\right) m(k) x_{1 n_{1}}(k)-\left(1-h_{3}(k)\right) m(k) D_{1} h_{1}(k)+\left(1-h_{3}(k)\right) m(k) D_{2} h_{2}(k)\right)\right] \text { holds and by }
\end{aligned}
$$


setting $\left.\phi=-\left[\left(1-h_{3}(k)\right) m(k) x_{1 n_{1}}(k)-\left(1-h_{3}(k)\right) m(k) D_{1} h_{1}(k)+\left(1-h_{3}(k)\right) m(k) D_{2} h_{2}(k)\right)\right]$, $\Delta \partial=\left[\begin{array}{l}\mathbf{0} \\ \mathbf{B}_{2}\end{array}\right] \in \mathbf{R}^{n \times 1}$, and $\delta_{x}=v x_{1 n_{1}}(k)+\phi \in R^{1 \times 1}$, it leads to $\Delta \mathbf{A}_{1}(k) \mathbf{x}(k)+\Delta \boldsymbol{\eta}=\left[\begin{array}{l}\mathbf{0} \\ \mathbf{B}_{2}\left(v x_{1 n_{1}}(k)+\phi\right)\end{array}\right]=\left[\begin{array}{l}\mathbf{0} \\ \mathbf{B}_{2}\end{array}\right]\left(v x_{1 n_{1}}(k)+\phi\right)=\Delta \partial \delta_{x}$.

To make the observer to be stable the eigenvalues of $\tilde{A}=\left[\begin{array}{cc}A_{1}-K_{4} C & -K_{3} \\ K_{2} C & K_{1}\end{array}\right]$ must be all in the unit circle. To make the observer robust to the general disturbances and sensitive to the fault, the following indicators of $H_{\infty, F}$ and $H_{-, F}$ need to be defined in eq. (18) and the indicator of $\frac{H_{\infty, F}}{H_{-, F}}$ should be minimized.

$$
\left\{\begin{array}{l}
H_{\infty, F}=\min \left(\max _{z=e^{j \omega}}\left\|\tilde{G}_{d^{*} r j}\left(\tilde{A}, K_{d}, z\right)\right\|_{F}, \omega \in[0,2 \pi)\right) \\
H_{-, F}=\max \left(\min _{z=e^{j \omega}}\left\|\tilde{G}_{f r}\left(\tilde{A}, K_{d}, z\right)\right\|_{F}, \omega \in[0,2 \pi)\right)
\end{array}\right.
$$

Where, \|\|$_{F}$ represents the Frobenius norm and this norm has the equivalent function of 2 norm. However, Frobenius norm is much easier to calculate.

In this study, since most of the faults are either an abrupt fault or a slow change fault their frequencies are close to zero when the faults are stable. However, the disturbances usually have higher frequencies. Therefore, we assume that the frequencies of the faults and the disturbances do not overlap with each other in this paper.

For the better restrain of the effect of the general disturbances to the residual the zeros assignment method has been used to determine parts of the feedback matrix. First, we need to know the definition of zeros in the system. Reference [22], [23], and [24] give the definition of zeros as follows.

$$
\begin{aligned}
& \mathbf{x}(k+1)=\mathbf{A x}(k)+\mathbf{B} \mathbf{u}(k) \\
& \mathbf{y}(k)=\mathbf{C x}(k)+\mathbf{D u}(k)
\end{aligned}
$$

Where $\mathbf{x} \in \mathbf{R}^{n \times 1}, \mathbf{u} \in \mathbf{R}^{r \times 1}, \mathbf{y} \in \mathbf{R}^{l \times 1}, \mathbf{A}, \mathbf{B}, \mathbf{C}, \mathbf{D}$ are the constant matrices with corresponding dimensions. Set $\operatorname{rank}(\mathbf{B})=r, \operatorname{rank}(\mathbf{C})=l$. Then the limited invariant zeros of transfer function $u(k)$ to $\mathbf{y}(k)$ are the complex set which satisfy this following requirement.

$$
\operatorname{rank}[P(z)]=\operatorname{rank}\left[\begin{array}{cc}
z \mathbf{I}-\mathbf{A} & -\mathbf{B} \\
\mathbf{C} & \mathbf{D}
\end{array}\right]<\min (r, l)+n .
$$

If $r=l$, then the limited invariant zeros are the zeros of the Eigenpolynomial as shown in eq. (20).

$$
Z=\operatorname{det}(P(z)) \text {. }
$$


By non-smooth robust observer in eq. (12) and according to the definition of zeros, the zeros of transfer function of the general disturbance $d^{*}(k)$ to residual $r(k)$ should be the complex numbers which satisfy the following condition.

$\operatorname{rank} \tilde{P}_{d^{*}}(z)<\min (1, r+2)+n+m$

Where $\tilde{P}_{d^{*}}(z)=\left[\begin{array}{cc}z \mathbf{I}-\tilde{\mathbf{A}} & -\tilde{\mathbf{B}}_{d^{*}} \\ \tilde{\mathbf{C}} & \mathbf{D}_{d^{*}}\end{array}\right]=\left[\begin{array}{ccc}z \mathbf{I}-\mathbf{A}_{l}+\mathbf{K}_{4} \mathbf{C} & \mathbf{K}_{3} & -\mathbf{B}_{d^{*}}^{*}+\mathbf{K}_{4} \mathbf{D}_{d^{*}} \\ -\mathbf{K}_{2} \mathbf{C} & z \mathbf{I}-\mathbf{K}_{1} & -\mathbf{K}_{2} \mathbf{D}_{d^{*}} \\ \mathbf{C} & \mathbf{0} & \mathbf{D}_{d^{*}}\end{array}\right]$.

The rank of matrix in eq. (22) is

$$
\begin{aligned}
& \operatorname{rank} \tilde{P}_{d^{*}}(z)=\operatorname{rank}\left[\begin{array}{ccc}
z \mathbf{I}-\tilde{\mathbf{A}} & -\tilde{\mathbf{B}}_{d^{*}} \\
\tilde{\mathbf{C}} & \mathbf{0}
\end{array}\right]=\operatorname{rank}\left[\begin{array}{ccc}
z I-\mathbf{A}_{l}+\mathbf{K}_{4} \mathbf{C} & -\mathbf{K}_{3} & -\mathbf{B}_{d^{*}}^{*}+\mathbf{K}_{4} \mathbf{D}_{d^{*}} \\
-\mathbf{K}_{2} \mathbf{C} & z \mathbf{I}-\mathbf{K}_{1} & -\mathbf{K}_{2} \mathbf{D}_{d^{*}} \\
\mathbf{C} & \mathbf{0} & \mathbf{D}_{d^{*}}
\end{array}\right] . \\
& =\operatorname{rank}\left[\begin{array}{ccc}
z \mathbf{I}-\mathbf{A}_{l}+\mathbf{K}_{4} \mathbf{C} & -\mathbf{K}_{3} & -\mathbf{B}_{d^{*}}^{*}+\mathbf{K}_{4} \mathbf{D}_{d^{*}} \\
\mathbf{0} & z \mathbf{I}-\mathbf{K}_{1} & \mathbf{0} \\
C & \mathbf{0} & \mathbf{0}
\end{array}\right] \\
& =\operatorname{rank}\left[\begin{array}{ccc}
z \mathbf{I}-\mathbf{A}_{l}+\mathbf{K}_{4} \mathbf{C} & -\mathbf{K}_{3} & -\mathbf{B}_{d^{*}}^{*}+\mathbf{K}_{4} \mathbf{D}_{d^{*}} \\
C & \mathbf{0} & \mathbf{0} \\
\mathbf{0} & z \mathbf{I}-\mathbf{K}_{1} & \mathbf{0}
\end{array}\right] \\
& =\operatorname{rank}\left[\begin{array}{ccc}
z \mathbf{I}-\mathbf{A}_{l}+\mathbf{K}_{4} \mathbf{C} & -B_{d^{*}}^{*}+K_{4} D_{d^{*}} & -K_{3} \\
C & 0 & \mathbf{0} \\
\mathbf{0} & \mathbf{0} & z \mathbf{I}-\mathbf{K}_{1}
\end{array}\right]
\end{aligned}
$$

By eq. (23), all the complex numbers of $\mathrm{z}$ which satisfy $\left|z \mathbf{I}-\mathbf{K}_{1}\right|=0$ are the limited invariant zeros of the transfer function $d^{*}(k)$ to $r(k)$. Therefore, we can calculate feedback matrix $\mathbf{K}_{1}$ to set certain zeros of the transfer function of $d^{*}(k)$ to $r(k)$. And these zeros we add in the system can block the effect of the disturbance to the residual.

According to reference [22], [23], and [24], we know that if the zeros are just equal to the input poles of the system, the input will be interrupted by the zeros and can not pass to the output end. Therefore, if we just set the zeros of the transfer function of the general disturbances to the residual at the major frequency points of the disturbances the effect of the disturbances to the residual will be reduced effectively.

If the major frequencies of the disturbances are $\omega_{i}(\mathrm{i}=1,2, \ldots, \mathrm{h}), \mathrm{h}$ is the number of major disturbances, and $T$ is the sampling time. The poles of the disturbances are $z_{i}=e^{ \pm\left(\omega_{i} T\right) j}$ and therefore the zeros should be set exactly at $z_{i}=e^{ \pm\left(\omega_{i} T\right) j}$. In reference [21], [22], and [23], they points out that for a single input and single output system (SISO) the frequency of the input will not change 
after pass through the system. Therefore, if we use the conventional non-robust observer to obtain the residual and analyze the residual's frequency spectrum, we can obtain the major disturbances' frequencies. If we set $\mathbf{K}_{1}$ as below

$$
\mathbf{K}_{1}=\left[\begin{array}{ccc}
\mathbf{k}_{1}^{\prime} & \mathbf{0} \ldots \ldots & \mathbf{0} \\
\mathbf{0} & \mathbf{k}_{2}^{\prime} \ldots \ldots & \mathbf{0} \\
& \ddots & \\
\mathbf{0} & \mathbf{0} \ldots \ldots & \mathbf{k}_{h}^{\prime}
\end{array}\right]=\operatorname{diag}\left(\mathbf{k}_{i}^{\prime}\right), \mathbf{k}_{i}^{\prime}=\left[\begin{array}{cc}
\cos \left(\omega_{i} T\right) & -\sin \left(\omega_{i} T\right) \\
\sin \left(\omega_{i} T\right) & \cos \left(\omega_{i} T\right)
\end{array}\right],(\mathrm{i}=1,2, \ldots, \mathrm{h}) .
$$

The zeros of the transfer function of the disturbances to residual will just be the same as the poles of the disturbances if $\mathbf{K}_{1}$ is designed according to eq. (24). In reference [25] and [26], the similar methods have been used to restrain the effect of the disturbances in fault detection. However, they focus on the smooth nonlinear system with continuous time.

Once $\mathbf{K}_{1}$ is designed according to eq. (29), the other feedback matrices of $\mathbf{K}_{2}, \mathbf{K}_{3}, \mathbf{K}_{4}$ should be calculated by solving a constrained optimization problem in eq. (25).

In fact, the ultimate purpose of design a robust observer is to select the other feedback matrices i.e., $\mathbf{K}_{2}, \mathbf{K}_{3}, \mathbf{K}_{4}$ to make the observer sensitive to the fault and insensitive to the disturbances. According to this purpose and the constrain of making sure the observer to be stable, the design of the robust observer can be concluded as an constrained optimization problem and it can be formulated in eq. (25).

$$
\begin{cases}\min & \left(\frac{H_{\infty, F}}{H_{-, F}}\right) \\ \text { s.t. } & |\lambda(\tilde{A})|<1\end{cases}
$$

In fact, according to eq. (18) $\frac{H_{\infty, F}}{H_{-, F}}$ is a function of $\mathbf{K}_{2}, \mathbf{K}_{3}, \mathbf{K}_{4}$ and the elements of $\mathbf{K}_{2}, \mathbf{K}_{3}, \mathbf{K}_{4}$ are the design variables. $\lambda($.$) represents calculating the eigenvalues of the matrix inside in the bracket$ and $|\lambda(\tilde{A})|<1$ means that the norm of all the eigenvalues of $\tilde{A}$ must be less than 1 . In other words, the constrain means that all the eigenvalues of $\tilde{A}$ must be inside the unit circle.

From eq. (19), it can be seen that the purpose of designing the robust observer is to minimize the norm of the transfer function of the residual to the general disturbances and maximize the norm of the transfer function of the residual to the fault. Therefore, the robust observer can restrain the effect of the general disturbances to the residual and amplify the effect of the fault to the residual. 


\section{Application}

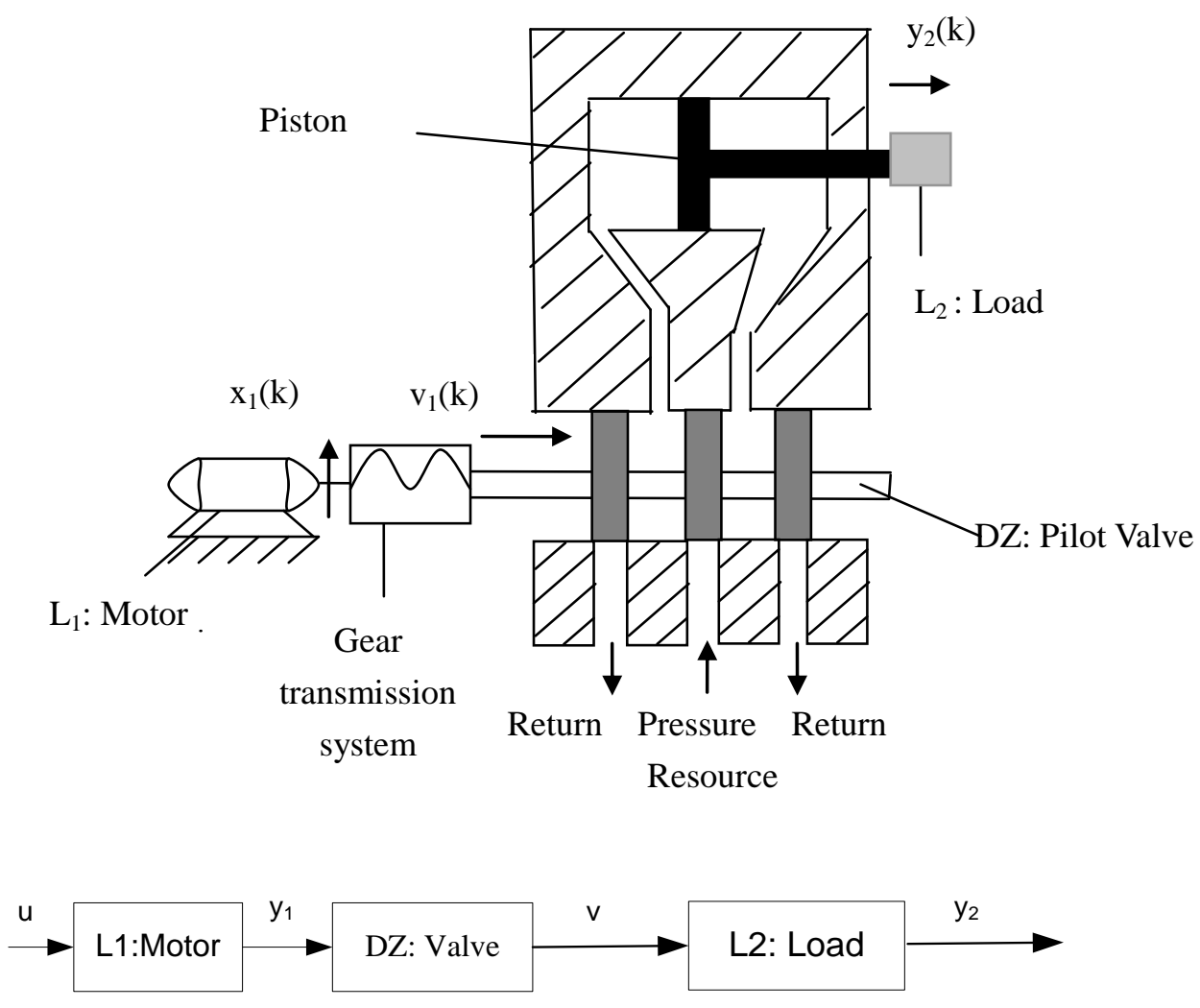

Fig.3 Motor servo hydraulic system

In Fig.3, the DC motor in this system can be regarded as a linear part represented by $\mathrm{L}_{1}$ and the load can be regarded as another linear system represented by $L_{2}$. The dynamic of the pilot valve whose overlapping on the fluid orifice causes a dead-zone represented by DZ. Therefore, the motor servo hydraulic system can be regarded as a sandwich system with dead zone. It should be notice that in fact due to the existence of the gears in the system backlash character will be existent. However, in this case, for simplification the backlash character has not been taken into consideration. This system is in many applications as a force amplifier. A smaller force is required to position the pilot valve, and a larger force is generated on the actuator piston. The sandwich system can be shown in eq. (26) and the parameters are derived from reference [3] with some modifications.

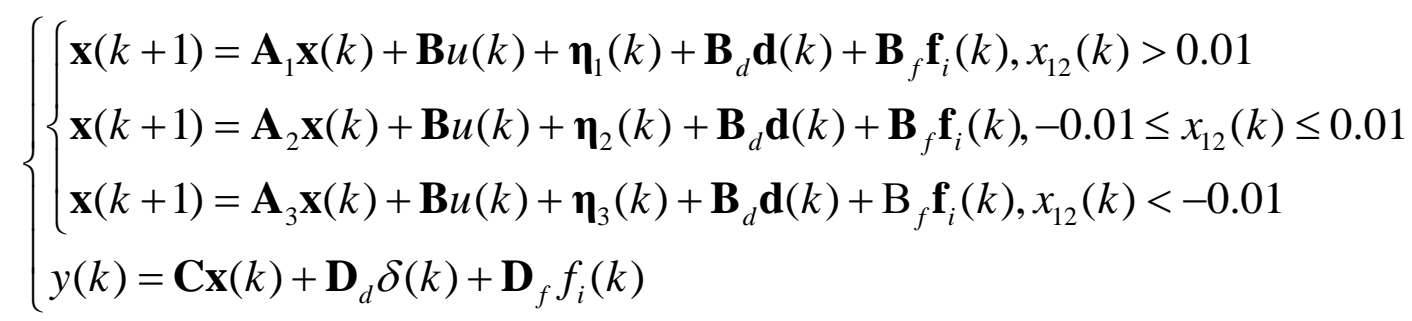

Where, 


$$
\begin{aligned}
& \mathbf{A}_{1}=\mathrm{A}_{3}=\left[\begin{array}{llcr}
0.82 & 0 & 0 & 0 \\
0.1 & 0.45 & 0 & 0 \\
0 & 0.25 & 0.85 & 0 \\
0 & 0 & 0.2 & 0.9
\end{array}\right], \mathbf{A}_{2}=\left[\begin{array}{cccc}
0.82 & 0 & 0 & 0 \\
0.1 & 0.45 & 0 & 0 \\
0 & 0 & 0.85 & 0 \\
0 & 0 & 0.2 & 0.9
\end{array}\right], \mathbf{B}=\left[\begin{array}{l}
0.4 \\
0 \\
0 \\
0
\end{array}\right], \quad \boldsymbol{\eta}_{1}=\left[\begin{array}{l}
0 \\
0 \\
-0.0025 \\
0
\end{array}\right], \\
& \boldsymbol{\eta}_{2}=\left[\begin{array}{l}
0 \\
0 \\
0 \\
0
\end{array}\right], \boldsymbol{\eta}_{3}=\left[\begin{array}{l}
0 \\
0 \\
0.0025 \\
0
\end{array}\right], \mathbf{C}=\left[\begin{array}{llll}
0 & 0 & 0 & 1
\end{array}\right] .
\end{aligned}
$$

The input $u(k)=6 \sin (6 T k)$ and the time of simulation is 20 seconds. The disturbances are shown as below.

$$
\mathbf{d}(k)=\left[\begin{array}{lll}
d_{1}(k) & d_{2}(k) & d_{3}(k)
\end{array}\right]^{T}=[0.2 \sin (20 \times T \times k), 0.4 \sin (20 \times T \times k), \operatorname{random}(-0.01,0.01)]^{T} \quad,
$$

$d_{1}(k)$ is the disturbance to the input $u(k)$ and $d_{2}(k)$ is the disturbance to the second linear part (disturbance to the load). $d_{3}(k)$ is the noise of the output and we assume it is the random series which range between +0.01 to -0.01 .

As it is shown in eq. (27) we assume that the abrupt actuator and sensor fault occurs between 400 and 600 sample point and between 1200 and 1400 sample point, respectively. We defined it as the first type of fault.

The first type of (abrupt) $\mathbf{f}_{1}(k)=\left[f_{11}(k) \quad f_{12}(k)\right]^{T}=\left\{\begin{array}{l}{[0,0]^{T}, \text { else }} \\ {[0.3,0]^{T}, 400 \leq k \leq 600} \\ {[0,-0.4]^{T}, 1200 \leq k \leq 1400}\end{array}\right.$

As it is shown in eq. (28) we assume that the slow change actuator and sensor fault occurs between 200 and 600 sample point and between 1000 and 1600 sample point, respectively. We defined it as the second type of fault. The second type of fault (slow change) is shown as below.

$$
\mathbf{f}_{2}(k)=\left[f_{21}(k) \quad f_{22}(k)\right]^{T}=\left\{\begin{array}{l}
{[0,0]^{T}, \text { else }} \\
{[0.003(k-200), 0]^{T}, 200 \leq k \leq 400} \\
{[0.6,0]^{T}, 400 \leq k \leq 600} \\
{[-0.003(k-600), 0]^{T}, 600 \leq k \leq 800} \\
{[-0.002(k-1000), 0]^{T}, 1000 \leq k \leq 1200} \\
{[0,-0.4], 1200 \leq k \leq 1400} \\
{[0,0.002(k-1400)]^{T}, 1400 \leq k \leq 1600}
\end{array}\right.
$$

Fig. 4 shows the model in which the disturbances, model uncertainties, and faults are all taken into consideration. The relative matrices are given as follows.

$\mathbf{B}_{d}=\left[\begin{array}{ll}\mathbf{E}_{1} & \mathbf{E}_{2}\end{array}\right]=\left[\begin{array}{lcc}0 & 0 & 0.4 \\ 0 & 0 & 0 \\ 0.25 & 0 & 0 \\ 0 & 0 & 0\end{array}\right], \mathbf{B}_{f}=\left[\begin{array}{ll}0.4 & 0 \\ 0 & 0 \\ 0 & 0 \\ 0 & 0\end{array}\right], \mathbf{D}_{d}=\left[\begin{array}{lll}0 & 0 & 1\end{array}\right], \mathbf{D}_{f}=\left[\begin{array}{ll}0 & 1\end{array}\right]$. The model 
uncertainties are $\Delta \mathbf{A}=\left[\begin{array}{l}\Delta \partial_{1} \\ \Delta \partial_{2} \\ \Delta \partial_{2} \\ \Delta \partial_{2}\end{array}\right]=\left[\begin{array}{llll}-0.05 & 0 & 0 & 0 \\ 0 & 0 & 0 & 0 \\ 0 & 0 & -0.05 & 0 \\ 0 & 0 & 0 & 0.07\end{array}\right], \Delta \mathbf{B}=\left[\begin{array}{l}\Delta b_{1} \\ \Delta b_{2} \\ \Delta b_{3} \\ \Delta b_{4}\end{array}\right]=\mathbf{0}$. The general disturbances matrices and vectors are shown as follows. $\mathbf{B}_{d}^{*}=\left[\begin{array}{cccccccc}1 & 0 & 0 & 0 & 0 & 0.4 & 0 & 0 \\ 0 & 1 & 0 & 0 & 0 & 0 & 0 & 0 \\ 0 & 0 & 1 & 0 & 0.25 & 0.25 & 0 & 0 \\ 0 & 0 & 0 & 1 & 0 & 0 & 0 & 0\end{array}\right], \mathbf{d}^{*}=\left[\begin{array}{l}\Delta \partial_{1} \mathbf{x}+\Delta b_{1} u \\ \Delta \partial_{2} \mathbf{x}+\Delta b_{2} u \\ \Delta \partial_{3} \mathbf{x}+\Delta b_{3} u \\ \Delta \partial_{4} \mathbf{x}+\Delta b_{4} u \\ \delta(k) \\ d_{1} \\ d_{2} \\ d_{3}\end{array}\right], \mathbf{D}_{d}^{*}=\left[\begin{array}{llllllll}0 & 0 & 0 & 0 & 0 & 0 & 0 & 1\end{array}\right] 。$

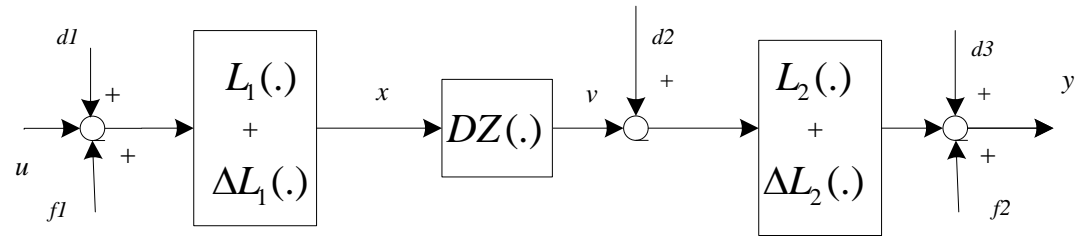

Fig.4 Structure of sandwich system with dead-zone with model uncertainty and disturbances
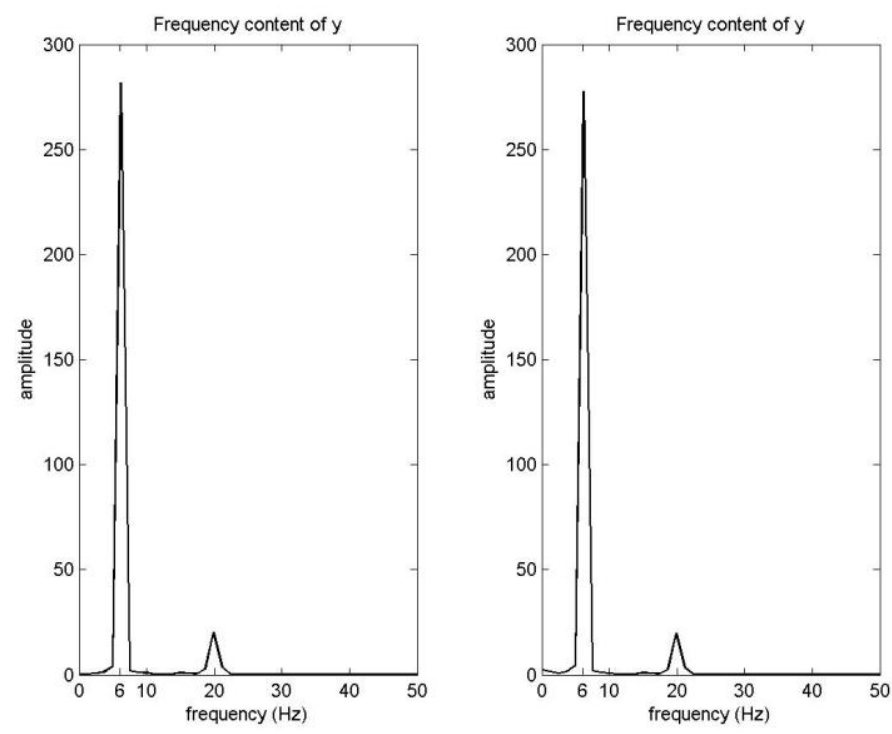

Fig.5 Spectrum of two types of fault residuals by non-robust observer

Without taking the robust into consideration, according to reference [21], the non-robust observer can be designed with the feedback matrix of $K_{f}=\left[\begin{array}{llll}0 & 0 & 0.1 & 0.1\end{array}\right]^{T}$ and the observer will converge by the observer convergence theorem in reference [22].

Fig.5 shows the residuals' frequency spectrum of non-robust observer of two types of faults. It is clear that the major energy of the residual consternated at two main frequencies points of $\omega_{1}=20 \mathrm{rad} / \mathrm{s}, \omega_{2}=6 \mathrm{rad} / \mathrm{s}$, respectively. $\mathrm{K}_{1}$ can be obtained by zeros assignment and other feedback 
matrices can be obtained by solving the constrained optimization problem in eq. (25).

$$
\begin{aligned}
& \mathbf{K}_{1}=\left[\begin{array}{llrc}
\cos (20 T) & -\sin (20 T) & 0 & 0 \\
\sin (20 T) & \cos (20 T) & 0 & 0 \\
0 & 0 & \cos (6 T) & -\sin (6 T) \\
0 & 0 & \sin (6 T) & \cos (6 T)
\end{array}\right] . \\
& \mathbf{K}_{1}=\left[\begin{array}{cccc}
0.9801 & -0.1987 & 0 & 0 \\
0.1987 & 0.9801 & 0 & 0 \\
0 & 0 & 0.9982 & -0.0600 \\
0 & 0 & 0.0600 & 0.9982
\end{array}\right], \quad \mathbf{K}_{2}=\left[\begin{array}{llll}
0.5330 & 0.2946 & 0.0306 & 0.1272
\end{array}\right]^{T} \text {, } \\
& \mathbf{K}_{3}=\left[\begin{array}{rrrr}
0.0804 & 0.7888 & 0.3808 & 0.6772 \\
0.7335 & 0.5405 & 0.2723 & -0.2127 \\
0.1766 & -0.1696 & 0.2486 & 0.0831 \\
0.2597 & 0.1348 & 0.2764 & -0.1084
\end{array}\right], \quad \mathbf{K}_{4}=\left[\begin{array}{llll}
0.4551 & 0.3975 & 0.8342 & 0.7044
\end{array}\right]^{T} \text {. }
\end{aligned}
$$
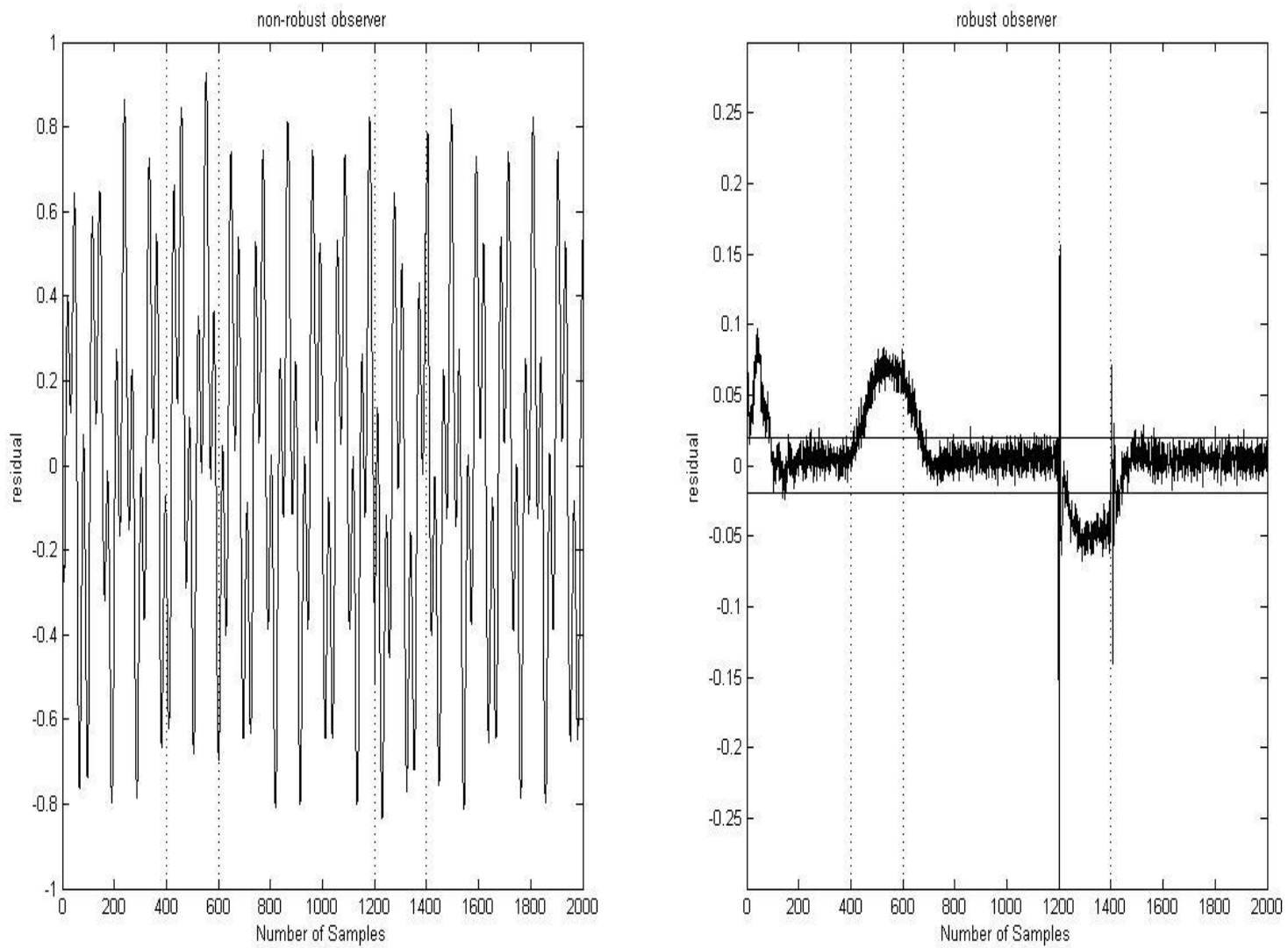

Fig.6 Residual comparison created by the first type of fault 

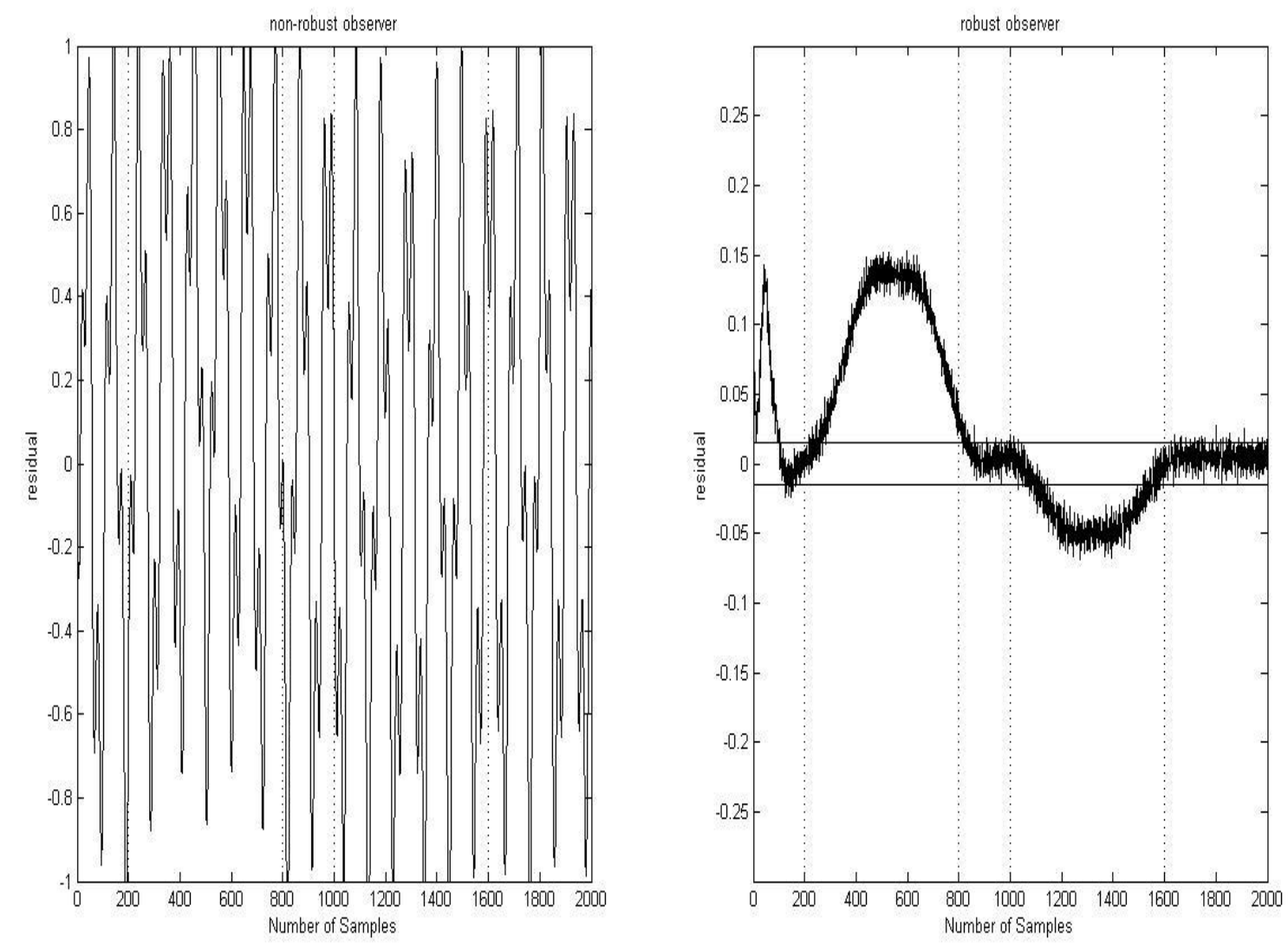

Fig.7 Residual comparison created by the second type of fault

From Fig.6 and Fig.7, for the non-robust observer when the first type and second type of faults occur the general disturbances are very strong and neither the actuator nor the sensor fault can be detected by the residual signal. On the contrary, the robust observer can detect these two types of faults more quickly and accurately comparing with the non-robust observer. When the abrupt actuator fault occurs between 400 and 600 sample points, the residual deviates from zero and gets back close to zero when no fault exists. When the abrupt sensor fault occurs between 1200 and 1400 sample points, the robust observer can detect this fault as well.

For the slow change fault i.e., second type of faults, from Fig. 7, it can be seen that the robust observer can detect the actuator fault when it occurs between 200 and 800 sample points and detect the sensor fault when it occurs between 1000 and 1600 sample points. However, the non-robust observer can not detect the fault since the residual caused by the disturbances suppressed the residual caused by the faults. Therefore, the robust observer has a much better performance than the non-robust one.

For further illustrating the effectiveness of reducing missing and false alarms of the robust observer, we discuss the missing alarm rate and false alarm rate of the observer. A threshold of $\mathrm{rt} 1= \pm 0.02$ and $\mathrm{rt} 2= \pm 0.02$ are set for the first type and second type of fault. When the residual is over the threshold fault alarm will be on otherwise fault alarm will be off. We define the fault missing alarm rate and false alarm rate as follows.

Missing alarm rate: 


$$
s_{l}=\frac{m_{l}}{m_{a}}
$$

$m_{l}$ : The number of sample points when the fault occurs while the residual is not over the threshold. $m_{a}:$ The total sample points of fault.

False alarm rate:

$$
s_{w}=\frac{m_{w}}{n_{a}}
$$

$m_{w}$ : The number of sample points which is over threshold while no fault actually occurs

$n_{a}$ : The sample points when there is no fault occurs.

In Fig.6 and Fig.7 the thresholds are represented by the two level solid lines. If the faults occur and their residuals are not over the threshold the missing alarms happen. On the contrary, if no faults occur and their residuals are over the threshold the false alarm happen. Since the initial states of the observer and the real system are not the same there is always a period of time for the observer to be stable and this time should be gotten rid of when calculating the missing and false alarm rates. For this reason, in the simulation the first 200 sample points have been gotten rid of. Finally, by using the non-smooth robust observer in the case of the first type of fault, the missing alarm rate is $10.75 \%$ and the false alarm rate is $9.21 \%$. For the second type of fault, the missing alarm rate is $15.42 \%$ and the false alarm rate is $13.17 \%$. For the non-robust observer, the missing and false alarm rates are almost close to $100 \%$.

\section{Conclusion}

Non-smooth sandwich systems with dead zone is a typical nonlinear non-smooth system which can be used to described many real systems in engineering such as DC motors, hydraulic servo systems, and precise moving stages. Thus, design a special observer which is especially good at detecting faults for this special system is of great importance in control engineering practice. By analysis the characteristics of the system, a general disturbance has been proposed by especially including the switching error caused by the system. Moreover, the conventional method of designing robust observer can be used by having this general disturbance. For further suppressing the influence of disturbances to the residual which will lead to false and missing alarm, the zero assignment method has been applied to find a suitable feedback matrix. The other feedback matrices can be found by solving an optimal problem in which the real purpose of the optimization is to reduce the effect of the disturbances to the residual and amplify the effect of the faults to the residual. By cleverly combining these methodologies 
together in this paper, a novel robust observer has been proposed which can restrain the influences of the general disturbance. In the future, the novel method may apply to other more complicated sandwich systems such as compound sandwich systems and parallel sandwich systems which can be used to describe other real engineering systems. The fault isolation and fault tolerant control of the sandwich systems is also a topic in our future research.

\section{Acknowledgement}

This work is partially supported by the National Science Foundation of China (61263016, 61371145, 61203108, 61171088, and 51165004), Nature and Science Foundation of Guangxi (2015GXNSFCA139019), the Innovation Program of Shanghai Municipal Education Commission (13YZ056), and the Science and Technology Commission of Shanghai (14140711200 and 14ZR1430300).

\section{References}

[1] J. Vörös, Recursive identification of Hammerstein systems with discontinuous nonlinearities containing dead-zones. IEEE Trans. Autom. Control.2003, 48(12):2203-2206.

[2] Y. Tan, R. Dong, R. Li. Recursive Identification of Sandwich Systems with Dead Zone and Application. IEEE Transactions on control systems technology, 2009, 17(4):945-951.

[3] A. Taware, G. Tao. Control of Sandwich Nonlinear System. Virginia: PhD Dissertation of University of Virginia, 2001: 27-37.

[4] M. Hou and R. J. Patton. An LMI approach to $H_{2} / H_{\infty}$ fault detection observers. Proc. UKACC Int. Conf. Control, 1996:305-310.

[5] W. Zhang et. al. Fault diagnosis based on RBF NN optimized by improved genetic algorithm in missile control system, Journal of Center South University (Science and Technology), 2011, 42(z1): 870-875.

[6] A. Alessandri, P. Coletta. Design of Luenberger observers for a class of hybrid linear systems. Hybrid Systems: Computation and Control HSCC'01, Berlin: Springer, 2001: 7-18.

[7] A. Alessandri, P. Coletta. Switching observers for continuous-time and discrete-time linear systems. Proceedings of the American Control Conference, Arlington: IEEE, 2001: 2516-2521.

[8] A. Juloski, W.P.M.H. Heemels, and S. Weiland. Observer design for a class of piecewise affine systems. Proceedings of the 41st IEEE Conference on Decision and Control, Las Vegas, USA: IEEE, 2002: 2606-2611.

[9] A. Juloski, W.P.M.H. Heemels, Y. Boers., F. Verschure. Two Approaches to State Estimation for a Class of Piecewise Affine Systems. Proceedings of the 41st IEEE Conference on Decision and Control, Las Vega, USA: IEEE, 2003: 143-148.

[10] B. Yan, H. Su, Wei Ma. Fault detection and identification for a class of nonlinear systems with model uncertainty. Applied Mathematical Modelling, 2016, 40: 7368-7381.

[11] P. Bahareh, M. Nader, K. Khashayar. Sensor Fault Detection, Isolation, and Identification Using Multiple-Model-Based Hybrid Kalman Filter for Gas Turbine Engines. IEEE Transactions on Control Systems and 
Technology, 24(4): 1184-1200.

[12] B. Park and S. Yoo. Fault detection and accommodation of saturated actuators for underactuated surface vessels in the presence of nonlinear uncertainties. Nonlinear Dynamic (2016) 85:1067-1077.

[13] J. and R. Patton. A new strategy for integration of fault estimation within fault-tolerant control. Automatica, 2016, 69:48-59.

[14] I. Boulaabi, A. Sellami, F. Hmida. Robust Delay-Derivative-Dependent Sliding Mode Observer for Fault Reconstruction : A Diesel Engine System Application. Circuits Syst Signal Process (2016) 35:2351-2372.

[15] F. Guo et.al. Fault detection for discrete-time Lipschitz nonlinear systems with signal-to-noise ratio constrained channels. Neurocomputing 2016, 194: 317-325.

[16] Z. Gao, X. Liu, M. Z. Q. Chen. Unknown Input Observer-Based Robust Fault Estimation for Systems Corrupted by Partially Decoupled Disturbances. IEEE Transactions on Industrial Electronics, 63(4): 2537-2547.

[17] B. Zhang et.al. A Novel Observer Design for Simultaneous Estimation of Vehicle Steering Angle and Sideslip Angle. IEEE Transactions on Industrial Electronics, 63(7): 4357-4366.

[18] Z. Zhou, Y.Tan. State-Estimation of Sandwich Systems with Dead Zone. Proceedings of the 2011 International Conference on Advanced Mechatronic Systems, Zhengzhou, China, August 11-13, 2011: 472-477.

[19] Z. Zhou, Y. Tan, R. Dong and H. He. Non-smooth Observer for Mechanical Systems Based on Sandwich Model with Backlash. ICIRA 2012, Part I, LNAI 7506, 2012: 611-621, 2012.

[20] Z. Zhou, Y. Tan. State Estimation of Sandwich Systems with Hysteresis Based on Non-smooth Observer. Control Theory \& Applications. 29(7), 2012: 847-856.

[21] Y. Tan, Z. Zhou, R. Dong and H. He. Fault Detection of Mechanical Systems with Inherent Backlash. 2013 10th IEEE International Conference on Networking, Evry, France Sensing and Control (ICNSC), 2013: 77-82.

[22] B. Liu Bao, W. Tang. Modern control theory ( $3^{\text {rd }}$ edition). Beijing: Press of Mechanical technology, 2006: 210-212.

[23] L. Wu. Theory of automatic control. Publisher of Tsinghua University, 1990: 68-96.

[24] S. Hu. Theory of automatic control ( $4^{\text {th }}$ edition). Publisher of National Defense, 1996.

[25] X. Dai, Z. Gao, T. Breikin, and H. Wang. Disturbance Attenuation in Fault Detection of Gas Turbine Engines: A Discrete Robust Observer Design. IEEE Transactions on Systems, Man, and Cybernetics-Part C: Applications and Reviews, 2009, 39(2): 234-239.

[26] X. Dai, T. Breikin, Z. Gao and H. Wang. Dynamic Modeling and Robust Fault Detection of a Gas Turbine Engine. 2008 American Control Conference Westin Seattle Hotel, Seattle, Washington, USA, 2008: 2160-2165. 